\title{
Benzathine Penicillin for Recurrence of Rheumatic Fever: The Jury Is Still Out
}

\author{
David A. Watkins · Bongani M. Mayosil
}

Published online: 27 November 2010

(C) Springer Science+Business Media, LLC 2010

We appreciate the recent investigation by Seckeler et al. [2] into the impact of secondary prophylaxis on the recurrence of acute rheumatic fever. Regrettably, the title of their study, which claims "no demonstrable effect of benzathine penicillin on recurrence of rheumatic fever" overstates their findings and could be misleading to clinicians and policymakers because two major study limitations were not addressed in their discussion.

First, "compliance" was defined as the proportion of benzathine penicillin G (BPG) doses delivered versus the number of doses expected over the duration of the followup period (it is unfortunate that the mean and median duration of the follow-up period is not given in this study). Thus in their study, a patient followed for 4 years could be $100 \%$ compliant for 3 years and $0 \%$ compliant for 1 year and still be classified as "compliant" while remaining at high risk for recurrence and potentially misclassified. It also is not surprising that their logistic regression did not find compliance to be associated with lower odds of recurrence. "One-unit" increases in compliance thus defined are, in our judgment, not clinically meaningful.

Second, as the authors rightly note, there is clinical equipoise as to the optimal dosing regimen for BPG. Although the 4-weekly intramuscular injection is considered to be the historical standard, a recent systematic review presents data that suggest otherwise. Dosing at 2-weekly intervals is shown to be significantly better at preventing recurrences than dosing at 4-weekly intervals, and compliance with more frequent dosing regimens is comparable with compliance with a 4-weekly regimen [1]. However, all the patients in the Seckeler et al. study received 4-weekly BPG, preventing assessment of any differential efficacy, effectiveness, or compliance related to 3- or 2-weekly dosing.

Thus, in addition to the noncontrolled, retrospective nature of the Seckeler et al. [2] study, we find insufficient evidence to conclude that BPG has no demonstrable effect on recurrence. Future studies should evaluate delivery methods and compliance in a more rigorous fashion.

\section{References}

1. Manyemba J, Mayosi BM (2003) Intramuscular penicillin is more effective than oral penicillin in secondary prevention of rheumatic fever: a systematic review. S Afr Med J 93:212-218

2. Seckeler MD, Hoke TR, Gurka MJ, Barton LL (2010) No demonstrable effect of benzathine penicillin on recurrence of rheumatic fever in Pacific island population. Pediatr Cardiol 31:849-852
D. A. Watkins $(\bowtie) \cdot$ B. M. Mayosil

Department of Medicine, Groote Schuur Hospital,

J Floor Old Main Building, Observatory 7925,

Cape Town, South Africa

e-mail: david.watkins@uct.ac.za 